\title{
The short-term effect of acupuncture on different ocular blood flow parameters in patients with primary open-angle glaucoma: a randomized, clinical study
}

This article was published in the following Dove Press journal:

Clinical Ophthalmology

\author{
Anna Leszczynska* \\ Lisa Ramm* \\ Eberhard Spoerl \\ Lutz E Pillunat \\ Naim Terai \\ Department of Ophthalmology, \\ University Hospital Dresden, Dresden, \\ Germany \\ *These authors contributed equally \\ to this work
}

Introduction: To investigate the effect of acupuncture on different ocular blood flow (OBF) parameters in patients with primary open-angle glaucoma (POAG).

Patients and methods: In a prospective, randomized study, 56 POAG patients were randomly assigned either to an eye-specific acupuncture (group I, $n=28$ ) or to an eye-unspecific acupuncture treatment (group II, $\mathrm{n}=28$ ). Blood flow parameters were measured before and 10 minutes after treatment. Parapapillary retinal blood flow was determined by the Heidelberg retina flowmeter. Retinal vessel diameters were measured by the dynamic vessel analyzer. Pulsatile ocular blood flow, ocular pulse amplitude, and IOP were investigated by the Langham OBF system. Systemic blood pressure was obtained additionally.

Results: Pulsatile ocular blood flow increased significantly after the eye-specific acupuncture treatment from $5.6 \pm 4.3$ to $6.7 \pm 4.9 \mu \mathrm{L} / \mathrm{min}(P=0.014)$. There was no significant change in parapapillary retinal blood flow, retinal vessel diameter, systemic blood pressure, or IOP after treatment in neither of the two groups.

Conclusion: An eye-specific acupuncture treatment may affect OBF in POAG patients.

Keywords: acupuncture, glaucoma, ocular blood flow, retinal vessel diameter, Heidelberg retina flowmeter, Langham ocular blood flow system, dynamic vessel analyzer

\section{Introduction}

Glaucomatous optic neuropathy is a multifactorial disease, which is characterized by remodeling of the optic nerve head and a progressive loss of retinal nerve fibers, leading to corresponding visual field defects. ${ }^{1}$

Intraocular pressure (IOP) is still the most important risk factor for glaucoma, and many therapeutic efforts have been made to lower IOP and to halt progression of this disease, either pharmacologically or by operative interventions. However, other factors than elevated IOP have been described, which seem to contribute to glaucomatous damage. Several studies identified disturbances of ocular blood flow (OBF) as a potential cause for visual field deterioration in glaucoma patients, despite a pharmacologically well-controlled IOP. ${ }^{2-4}$ Therefore, therapeutic options to treat OBF alterations in glaucoma patients have gained more and more interest in the last years. ${ }^{5}$ However, therapeutic alternatives and their beneficial effects to affect blood flow disturbances positively are limited and not sufficiently proven by the current data. ${ }^{6}$

In the field of the so-called alternative or complimentary therapies, it has been proposed that acupuncture, which belongs to the old Chinese traditional medicine
Correspondence: Naim Terai

Department of Ophthalmology,

University Hospital Dresden,

Fetscherstreet 74, 01307

Dresden, Germany

Tel +49 35| 458509 I

Fax +49 35। 4584335

Email naim.terai@uniklinikum-dresden.de 
and has been used for over 5,000 years now, might represent an alternative therapy in some glaucoma patients. ${ }^{6-8}$ In fact, some previous studies reported beneficial effects of acupuncture in terms of reducing IOP ${ }^{9,10}$ or improving visual acuity. ${ }^{8,11}$ Despite these results, there is still a lack of reliable, randomized data to evaluate the validity of acupuncture as a potential therapy form in glaucoma patients. Also, to the best of (our) knowledge, there are hardly any data on the effects of acupuncture on OBF parameters but quite a few studies on IOP changes after this treatment.

Therefore, the aim of the present randomized study was to investigate the effect of an "eye-specific" acupuncture treatment compared to an "eye-unspecific" acupuncture treatment on different OBF parameters in a cohort of age- and gender-matched glaucoma patients.

\section{Patients and methods}

\section{Patients}

Fifty-six patients with primary open-angle glaucoma (POAG) were included in this prospective, randomized study. The present study was registered (DRKS-ID DRKS00014565) according to the guidelines of ICMJE (International Committee of
Medical Journal Editors). All patients were recruited from the Department of Ophthalmology, University Hospital Carl Gustav Carus, Dresden, between 2014 and 2015. The study protocol was in agreement with the tenets of the Declaration of Helsinki and approved by the local ethics committee of the University of Dresden. All subjects signed a written informed consent before participation. Glaucoma was defined from optic nerve head damage and visual field damage typical in glaucoma patients. All glaucoma patients were under topical antiglaucoma medication. Exclusion criteria in glaucoma patients were as follows: topical therapy with dorzolamide, diabetes, age-related macular degeneration and other ocular diseases, previous intraocular surgeries and/or retinal laser photocoagulation, ocular injuries, or lens/corneal opacities, and smokers. Patients with migraine, epilepsy, uncontrolled arterial hypertension, and prior ( $<4$ weeks) steroid therapy, as well as pregnant/breastfeeding women were also excluded from the study. After meeting the eligibility criteria, participants were assigned to an eye-specific acupuncture treatment (group I) or an eye-unspecific acupuncture scheme (group II) after block randomization (Figure 1). The duration of glaucoma disease, the extent of glaucomatous damage,

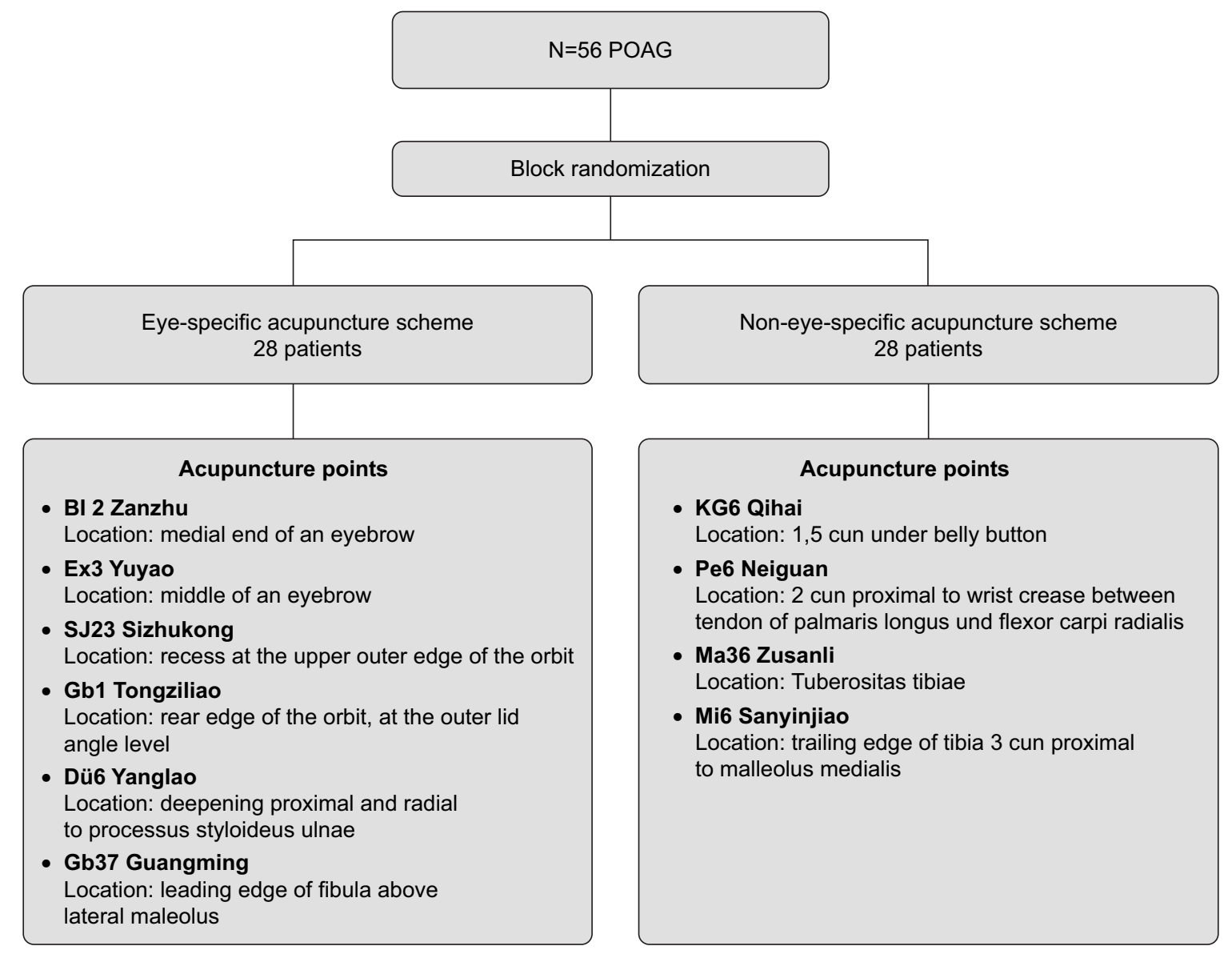

Figure I Needling points in the eye-specific acupuncture scheme and the non-eye-specific acupuncture scheme in POAG patients. Abbreviation: POAG, primary open-angle glaucoma. 
and the amount of topical antiglaucoma medications were comparable in both the groups and not significantly different. Before acupuncture, all patients underwent a complete ophthalmological examination of the anterior and the posterior segment. After mydriasis with tropicamid-1 \% (Mydrum, $5 \mathrm{mg} / 1 \mathrm{~mL}$; Chauvin Ankerpharm, Berlin, Germany), patients were instructed to sit for 10 minutes to achieve stable hemodynamic conditions. Blood pressure was measured and blood flow measurements started with the Heidelberg retina flowmeter (HRF), followed by the OBF system and the dynamic vessel analyzer (DVA). Measurements were taken only in one eye of each patient. After completion of all measurements, acupuncture was performed in the same sitting position.

\section{Acupuncture treatment}

After disinfection (Desdermann pure, Schuelke, Norderstedt, Germany) of the skin, acupuncture treatment was performed with disposable stainless steel needles (Seirin B-type) by an experienced, licensed acupuncturist. Needling points in both the groups are displayed in Figures 1 and 2. These were chosen according to previous studies by Litscher et al who demonstrated eye-related changes in blood flow of nearby arteries after a specific acupuncture treatment scheme. ${ }^{12-14}$ Ten minutes after acupuncture, abovementioned blood flow measurements were repeated in the same manner with the same devices.

\section{Methods}

The following devices were used for blood flow measurements:

\section{HRF}

The HRF allows a noninvasive measurement of the parapapillary retinal blood flow. ${ }^{15}$ The system is based on the principles of the optic Doppler. It combines the laser Doppler technique with a confocal laser scanning system. Details of this method are described elsewhere. ${ }^{16}$ Briefly, a coherent infrared laser with a wavelength of $780 \mathrm{~nm}$ and a power of $180 \mu \mathrm{W}$ is applied on the retinal surface and the capillary perfusion is measured using the frequency shift of the moving erythrocytes. A twodimensional perfusion map is generated from the interpolation shifts. Relative blood volumes and relative velocities can be measured, and the relative flow is then calculated from these variables. In the present study, the mean value of three images was calculated for further analysis. The perfusion maps were analyzed by an updated AFFPIA (Automatic Full Field Perfusion Image Analyzer) software. ${ }^{17}$
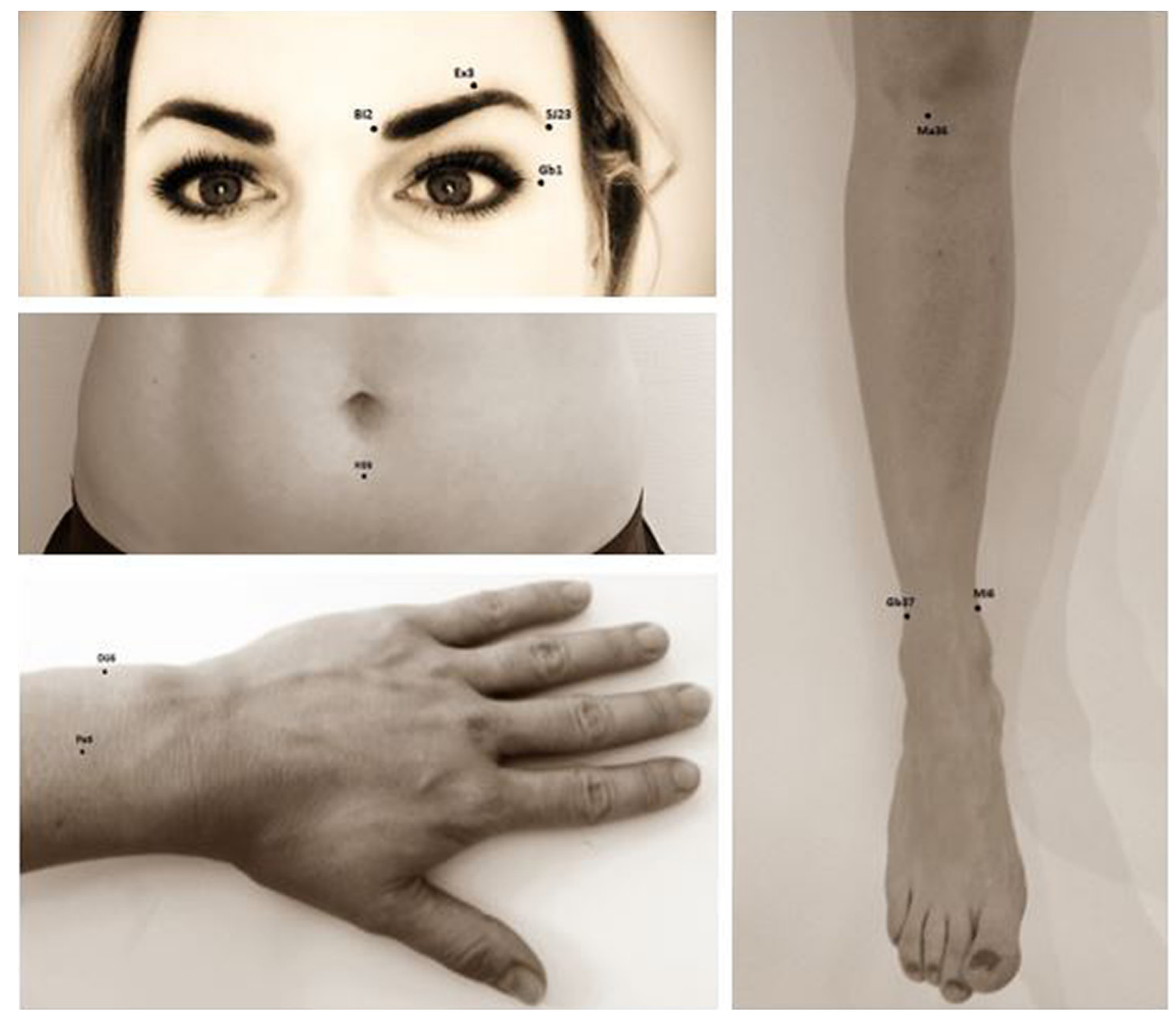

Figure 2 Illustration of needling points in the eye-specific acupuncture scheme and the eye-unspecific acupuncture scheme in primary open-angle glaucoma patients. 


\section{DVA}

The DVA is a commercially available system that comprises a fundus camera (Zeiss FF 450; Imedos Systems, Jena, Germany), a video camera, a high-resolution recorder, a real-time monitor, and a personal computer with vessel diameter analyzing software. The DVA allows the precise determination of retinal vessel diameter with a time resolution of 25 readings/sec. Retinal irradiance was $\sim 220 \mu \mathrm{W} \times \mathrm{cm}^{-2}$, which is $\sim 50$ times lower than the maximum level allowed for constant illumination of the retina at the wavelengths mentioned below. The system provides excellent reproducibility and sensitivity. ${ }^{18,19}$ In the present study, a major temporal arteriole and venule was studied. Measurement of retinal vessel diameters was taken between 1 and 2 disc diameters from the margin of the optic disc.

\section{OBF flowmeter}

The OBF (OBF system 3000; OBF Labs, Malmesbury, Wiltshire, UK) was used for the noninvasive measurement of the ocular pulse amplitude, the pulsatile OBF, and the IOP. The system represents a pneumotonometer. Principles of this measurement device are described elsewhere. ${ }^{20,21}$ Briefly, the OBF records the continuous change in the IOP pulse wave with each heart beat at the moment blood enters the eye. The ocular pulse amplitude (in $\mathrm{mmHg}$ ) is described as the difference between the maximum and the minimum IOP. The pulsatile ocular blood flow (POBF) (in $\mu \mathrm{L} / \mathrm{min}$ ) is automatically calculated by the software of the instrument on the basis of ocular pulse amplitude.

\section{Statistical data analysis}

Statistical data analysis was done using the software SPSS (version 17.0, Chicago, IL, USA). All variables were normally distributed according the Kolmogorov-Smirnov test. To detect changes in different blood flow parameters before and after acupuncture, an analysis of variance with repeated measurements was used in both the groups. A $P$-value $<0.05$ was considered to be statistically significant. All values are given as the mean $\pm \mathrm{SD}$.

\section{Results}

In the present prospective study, 56 glaucoma patients were included and randomly assigned either to an eye-specific (group I) or to an eye-unspecific (group II) acupuncture scheme. In both the groups, gender distribution was not significantly different $(P=0.6)$. Mean age in group I was $50.5 \pm 9.82$ years (range: $33-69$ years) and in group II was $48.7 \pm 9.7$ years (range: $32-65$ years), with no statistically
Table I Baseline and clinical data in group I and group II

\begin{tabular}{|c|c|c|c|}
\hline & Group I & Group II & $P$-value \\
\hline Age (years) & $50.5 \pm 9.82$ & $48.7 \pm 9.7$ & 0.608 \\
\hline Visual acuity (decimal score) & $0.90 \pm 0.18$ & $0.96 \pm 0.07$ & 0.457 \\
\hline RR syst_before $(\mathrm{mmHg})$ & $|28.9| \pm|| .28$ & $129.26 \pm \mid 1.58$ & 0.882 \\
\hline RR syst_after (mmHg) & $125.0 \pm 13.73$ & $126.3 \pm 10.7$ & 0.594 \\
\hline RR diast_before (mmHg) & $82.17 \pm 6.18$ & $8 I .1 I \pm 5.43$ & 0.527 \\
\hline RR diast_after $(\mathrm{mmHg})$ & $80.87 \pm 6.68$ & $80.56 \pm 6.1$ & 0.853 \\
\hline IOP (mmHg) & $|6.7| \pm 2.26$ & $15.45 \pm 4.97$ & 0.477 \\
\hline$M D(d B)$ & $-2.19 \pm 2.0$ & $-2.57 \pm 2.8 \mathrm{I}$ & 0.726 \\
\hline PSD (dB) & $2.87 \pm 2.57$ & $2.65 \pm 1.93$ & 0.481 \\
\hline Topical antiglaucoma medication & $1.55 \pm 1.29$ & $1.0 \pm 1.11$ & 0.28 \\
\hline
\end{tabular}

Note: Group I, eye-specific acupuncture; group II, eye-unspecific acupuncture group. Abbreviations: IOP, intraocular pressure; MD, mean deviation; PSD, pattern standard deviation; RR diast, diastolic blood pressure; RR syst, systolic blood pressure.

significant difference between the groups $(P=0.608)$. Baseline and clinical data are given in Table 1 and were not significantly different between the two groups.

\section{HRF}

There was no significant change in parapapillary retinal blood flow (volume, flow, and velocity) before or after acupuncture in neither of the groups (Table 2):

Mean volume (arbitrary unit, AU) in group I was 15.9 \pm 6.4 AU before acupuncture and 14.9 $\pm 3.4 \mathrm{AU}$ after acupuncture $(P=0.365)$. Mean flow in group I was $271.0 \pm 159.1 \mathrm{AU}$ before acupuncture and 247.3 $\pm 84.0 \mathrm{AU}$ after acupuncture $(P=0.446)$. Mean velocity $(\mathrm{kHz})$ in group I was $0.9 \pm 0.5 \mathrm{kHz}$ before acupuncture and $0.8 \pm 0.3 \mathrm{kHz}$ after acupuncture $(P=0.628)$.

Mean volume in group II was $16.3 \pm 6.2 \mathrm{AU}$ before acu-

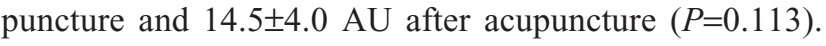
Mean flow in group II was 274.6 \pm 160.2 AU before acupuncture and 224.5 $\pm 86.0 \mathrm{AU}$ after acupuncture $(P=0.119)$. Mean velocity $(\mathrm{kHz})$ in group I was $0.9 \pm 0.5 \mathrm{kHz}$ before acupuncture and $0.7 \pm 0.3 \mathrm{kHz}$ after acupuncture $(P=0.153)$.

\section{DVA}

There was no significant change in retinal vessel diameters before or after acupuncture in neither of the groups (Table 2):

Mean diameter of the venules in group I was $134 \pm 17.8 \mu \mathrm{m}$ before acupuncture and $135.7 \pm 19 \mu \mathrm{m}$ after acupuncture $(P=0.086)$. Mean diameter of the arterioles in group I was $102.5 \pm 14.9 \mu \mathrm{m}$ before acupuncture and $100.9 \pm 18 \mu \mathrm{m}$ after acupuncture $(P=0.319)$.

Mean diameter of the venules in group II was $134.5 \pm 19.8 \mu \mathrm{m}$ before acupuncture and $135.5 \pm 19.8 \mu \mathrm{m}$ after acupuncture $(P=0.22)$. Mean diameter of the arterioles in group II was 
Table 2 Ocular blood flow parameters before and after acupuncture in group I (eye-specific acupuncture group) and group II (eyeunspecific acupuncture group)

\begin{tabular}{|c|c|c|c|c|c|c|}
\hline Parameters & $\begin{array}{l}\text { Group I } \\
\text { (before } \\
\text { acupuncture) }\end{array}$ & $\begin{array}{l}\text { Group I } \\
\text { (after } \\
\text { acupuncture) }\end{array}$ & $P$-value & $\begin{array}{l}\text { Group II } \\
\text { (before } \\
\text { acupuncture) }\end{array}$ & $\begin{array}{l}\text { Group II } \\
\text { (after } \\
\text { acupuncture) }\end{array}$ & $P$-value \\
\hline \multicolumn{7}{|l|}{ HRF } \\
\hline - Mean volume $(A U) \pm S D$ & $15.9 \pm 6.4$ & $14.9 \pm 3.4$ & 0.365 & $16.3 \pm 6.2$ & $14.5 \pm 4.0$ & 0.113 \\
\hline - Mean flow $(A U) \pm S D$ & $27 I \pm 159.1$ & $247.3 \pm 84$ & 0.446 & $274.6 \pm 160.2$ & $224.5 \pm 86.0$ & 0.119 \\
\hline - Mean velocity $(\mathrm{kHz}) \pm \mathrm{SD}$ & $0.9 \pm 0.5$ & $0.8 \pm 0.3$ & 0.628 & $0.9 \pm 0.5$ & $0.7 \pm 0.3$ & 0.153 \\
\hline \multicolumn{7}{|l|}{ DVA } \\
\hline - Mean diameter of venules $(\mu \mathrm{m}) \pm S D$ & $134 \pm 17.8$ & $135.7 \pm 19$ & 0.086 & $134.5 \pm 19.8$ & $135.5 \pm 19.8$ & 0.220 \\
\hline - Mean diameter of arterioles $(\mu \mathrm{m}) \pm S D$ & $102.5 \pm 14.9$ & $100.9 \pm 18$ & 0.319 & $104.3 \pm 14.8$ & $104.8 \pm 15.1$ & 0.574 \\
\hline \multicolumn{7}{|l|}{ OBF } \\
\hline - IOP (mmHg) & $16.1 \pm 4.5$ & $15.3 \pm 5.7$ & 0.378 & $16.4 \pm 4.5$ & $16.5 \pm 4.8$ & 0.881 \\
\hline - Pulse amplitude (mmHg) & $3.4 \pm 1.0$ & $3.7 \pm 1.5$ & 0.204 & $4.3 \pm 1.9$ & $4.4 \pm 2.1$ & 0.996 \\
\hline - Pulsatile OBF ( $\mu \mathrm{L} / \mathrm{min})$ & $5.6 \pm 4.3$ & $6.7 \pm 4.9$ & 0.014 & $6.3 \pm 3.6$ & $6.8 \pm 4.7$ & 0.370 \\
\hline
\end{tabular}

Note: Statistically significant value is indicated in bold.

Abbreviations: AU, arbitrary unit; DVA, dynamic vessel analyzer; HRF, Heidelberg retina flowmeter; IOP, intraocular pressure; OBF, ocular blood flowmeter.

104.3 $\pm 14.8 \mu \mathrm{m}$ before acupuncture and $104.8 \pm 15.1 \mu \mathrm{m}$ after acupuncture $(P=0.574)$.

\section{OBF}

In group I, OBF-IOP was $16.1 \pm 4.5 \mathrm{mmHg}$ before acupuncture and $15.3 \pm 5.7 \mathrm{mmHg}$ after acupuncture $(P=0.378)$. Pulse amplitude was $3.4 \pm 1.0 \mathrm{mmHg}$ before acupuncture and $3.7 \pm 1.5 \mathrm{mmHg}$ after acupuncture $(P=0.204)$. POBF changed significantly from $5.6 \pm 4.3$ to $6.7 \pm 4.9 \mu \mathrm{L} / \mathrm{min}$ after acupuncture $(P=0.014)$ (Figure 3; Table 2).

In group II, OBF-IOP was $16.4 \pm 4.5 \mathrm{mmHg}$ before acupuncture and $16.5 \pm 4.8 \mathrm{mmHg}$ after acupuncture $(P=0.881)$. Pulse amplitude was $4.3 \pm 1.9 \mathrm{mmHg}$ before acupuncture and $4.4 \pm 2.1 \mathrm{mmHg}$ after acupuncture $(P=0.996)$. POBF changed insignificantly from $6.3 \pm 3.6$ to $6.8 \pm 4.7 \mu \mathrm{L} / \mathrm{min}(P=0.370)$.

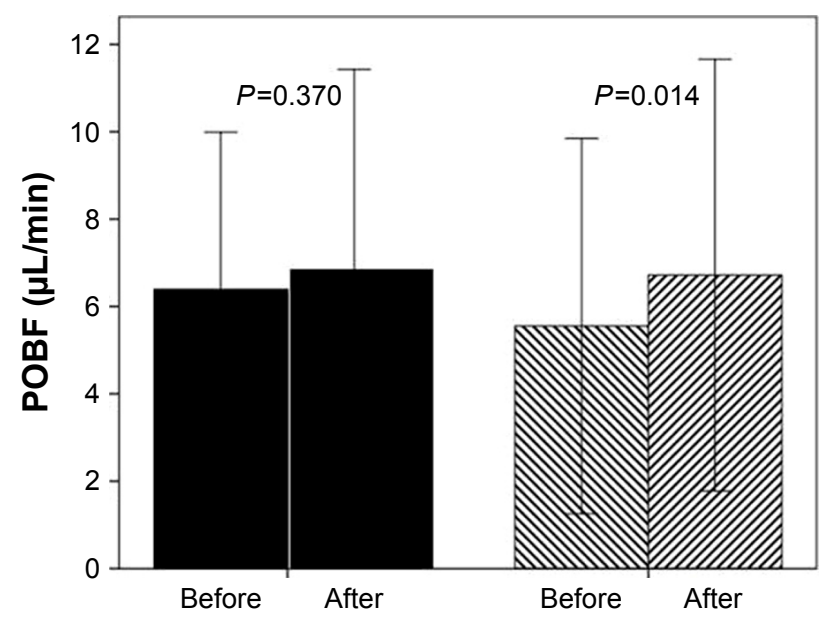

Figure $3 \mathrm{POBF}(\mu \mathrm{L} / \mathrm{min})$ in the eye-unspecific acupuncture group (black column) and in the eye-specific acupuncture group (shaded column).

Abbreviation: POBF, pulsatile ocular blood flow.

\section{Systemic blood pressure}

Systemic blood pressure and pulse rate were not different before acupuncture and showed no statistically significant change after acupuncture in neither of the two groups (Table 1).

\section{Discussion}

In the present study, the short-term effect of an eye-specific acupuncture treatment vs an eye-unspecific acupuncture treatment regimen on different OBF parameters was investigated in 56 glaucoma patients. Acupuncture needling points were chosen according to Litscher et al who described marked increases in blood flow in different regions of the body, presumably the brain and the eye, after specific acupuncture regimens. ${ }^{12-14}$ Hence, he provided evidence that a specific acupuncture also produces a specific reproducible and quantifiable effect on blood flow in the arteries of the brain and the eye. The eye-unspecific needling points were also chosen according to study results of Litscher et al where no effect on ocular perfusion was observed. ${ }^{12-14}$

We were able to show that the eye-specific treatment affected choroidal blood flow in terms of an increase in POBF using the Langham OBF. However, parameters of retinal perfusion (parapapillary retinal blood flow and retinal vessel diameter), as measured by the HRF and the retinal vessel analyzer, were not affected by acupuncture in neither of the two groups. Our results are in part consistent with the findings of a recent study by Takayama et al. ${ }^{22}$ They investigated blood flow changes during acupuncture using the ultrasound color Doppler (retrobulbar blood flow) and observed a significant decrease in the vascular resistance of the short posterior ciliary arteries resulting in an increase in blood flow to the 
choroid. Based on previous findings, the authors postulated a sympathetic and parasympathetic blood flow regulation via a release of nitric oxide or calcitonin gene-related peptide. ${ }^{22-24}$ However, the authors assumed that the regulation of the regional blood flow by a somatic afferent stimulation might be related to a somato-autonomic reflex mechanism in the choroidal blood flow of the eye. In a further experiment, the authors of the same study investigated the effect of acupuncture on the retrobulbar circulation in POAG patients. They detected a decrease of the distal vascular resistance in the central retinal artery and the short posterior ciliary artery indicating an increase of blood flow in the retina and the choroid. The increase of the retinal blood flow contradicts our study results because we did not measure a significant change of parapapillary retinal blood flow or retinal vessel diameter, which are two parameters characterizing retinal blood flow. However, differences to our results might arise for example from the location of the needling points. In the study by Takayama et al, needling point "LR3" was located on the foot of the patients. In our study, needling points of the eye-specific acupuncture scheme were located only in the facial region according to Litscher et al. ${ }^{14}$ Also, blood flow measurements were performed during needling stimulation and not 10 minutes after acupuncture treatment like in our study. Next, measurement techniques in both studies were completely different (color Doppler vs HRF/DVA), so a comparability of both studies is limited. To the best of (our) knowledge, there are so far no further studies investigating the effect of acupuncture on OBF parameters.

However, several previous studies performed IOP measurements before and after acupuncture. ${ }^{8-11}$ In our study, IOP was not significantly reduced after treatment, which contradicts recent study results by Law et al. ${ }^{7}$ They detected a slight but significant increase in mean IOP immediately after treatment session in both the groups. The authors assumed a temporary effect of acupuncture on IOP because diurnal IOP changes were not significant. However, due to differences in methodology (eg, acupuncture points, tonometry device, amount of acupuncture treatments, and needling technique), a direct comparison to our results is not allowed point by point. In extrapolation, several other studies reported IOP-lowering effects after acupuncture, ${ }^{8-11}$ which could not be confirmed by the results of the present study.

\section{Limitations}

Some limitations of this study have to be mentioned. As the incidence of glaucoma rises with increasing age, ${ }^{25}$ an untypical young patient group was included in this study. This is caused by the fact that only subjects without serious systemic diseases, such as uncontrolled hypertension and arteriosclerosis, should be included. Because of a well-known rising incidence of these diseases with age, ${ }^{26}$ recruitment of older POAG patients would have been difficult. Furthermore, due to ethical issues, IOP-lowering treatment (except from dorzolamide) was not interrupted. For this reason, a small influence of the topical therapy on the OBF cannot be ruled out with certainty. ${ }^{27}$

\section{Conclusion}

Taking together, the preliminary results of the present study showed a change in choroidal blood flow 10 minutes after a single eye-specific acupuncture treatment in glaucoma patients. This result might indicate a stimulatory effect of acupuncture on the autonomic nervous system via somatic nerves. ${ }^{22}$ Furthermore, especially crossover studies are needed to elucidate the duration of the stimulatory effect, the longitudinal impact on glaucoma disease, and a possible clinical relevance of this treatment. To do so, larger sample sizes and more standardized and homogenous (also repeated) acupuncture regimens are needed to provide adequate comparability and to evaluate the validity of this method as a potential complimentary therapy in glaucoma patients.

\section{Disclosure}

The authors report no other conflicts of interest in this work.

\section{References}

1. Quigley HA, West SK, Munoz B, Mmbaga BB, Glovinsky Y. Examination methods for glaucoma prevalence surveys. Arch Ophthalmol. 1993;111(10):1409-1415.

2. Resch H, Garhofer G, Fuchsjäger-Mayrl G, Hommer A, Schmetterer L. Endothelial dysfunction in glaucoma. Acta Ophthalmol. 2009;87(1):4-12. Epub 2008 May 27.

3. Konieczka K, Choi HJ, Koch S, Fankhauser F, Schoetzau A, Kim DM. Relationship between normal tension glaucoma and Flammer syndrome. Epma J. 2017;8(2):111-117.

4. Fuchsjäger-Mayrl G, Wally B, Georgopoulos M, et al. Ocular blood flow and systemic blood pressure in patients with primary open-angle glaucoma and ocular hypertension. Invest Ophthalmol Vis Sci. 2004;45(3): 834-839.

5. Rom E. Sensory stimulation for lowering intraocular pressure, improving blood flow to the optic nerve and neuroprotection in primary open-angle glaucoma. Acupunct Med. 2013;31(4):416-421. Epub 2013 Sep 5.

6. Law SK, Li T. Acupuncture for glaucoma. Cochrane Database Syst Rev. 2013;31(5):CD006030.

7. Law SK, Lowe S, Law SM, Giaconi JA, Coleman AL, Caprioli J. Prospective Evaluation of Acupuncture as Treatment for Glaucoma. Am J Ophthalmol. 2015;160(2):256-265. Epub 2015 Apr 30.

8. Kurusu M, Watanabe K, Nakazawa T, et al. Acupuncture for patients with glaucoma. Explore (NY). 2005;1(5):372-376.

9. Ralston NS. Successful treatment and management of acute glaucoma using acupuncture. Am J Acupuncture. 1977;5(3):283-285.

10. Dabov S, Goutoranov G, Ivanova R, Petkova N. Clinical application of acupuncture in ophthalmology. Acupunct Electrother Res. 1985;10(1-2): 79-93.

11. Wong S, Ching R. The use of acupuncture in ophthalmology. Am J Chin Med. 1980;8(1-2):104-153. 
12. Litscher G, Wang L, Yang NH, Schwarz G. Computer-controlled acupuncture. Quantification and separation of specific effects. Neurol Res. 1999;21(6):530-534.

13. Litscher G, Wang L, Yang NH, Schwarz G. Ultrasound-monitored effects of acupuncture on brain and eye. Neurol Res. 1999;21(4):373-377.

14. Litscher G, Yang NH, Schwarz G, Wang L. [Computer-controlled acupuncture. A new construction for simultaneous measurement of blood flow velocity of the supratrochlear and middle cerebral arteries]. Biomed Tech (Berl). 1999;44(3):58-63. German.

15. Holló G. Scanning laser Doppler flowmeter study of retinal and optic disk blood flow in glaucomatous patients. Am J Ophthalmol. 1997; 123(6):859-860.

16. Michelson G, Schmauss B, Langhans MJ, Harazny J, Groh MJ, Principle GMJ. Principle, validity, and reliability of scanning laser Doppler flowmetry. J Glaucoma. 1996;5(2):99-105.

17. Michelson G, Welzenbach J, Pal I, Harazny J. Automatic full field analysis of perfusion images gained by scanning laser Doppler flowmetry. Br J Ophthalmol. 1998;82(11):1294-1300.

18. Polak K, Dorner G, Kiss B, et al. Evaluation of the Zeiss retinal vessel analyser. Br J Ophthalmol. 2000;84(11):1285-1290.

19. Garhofer G, Bek T, Boehm AG, et al. Ocular Blood Flow Research Association. Use of the retinal vessel analyzer in ocular blood flow research. Acta Ophthalmol. 2010;88(7):717-722.
20. Butt Z, O'brien C, O'brien C. Reproducibility of pulsatile ocular blood flow measurements. J Glaucoma. 1995;4(3):214-218.

21. Yang YC, Hulbert MF, Batterbury M, Clearkin LG. Pulsatile ocular blood flow measurements in healthy eyes: reproducibility and reference values. J Glaucoma. 1997;6(3):175-179.

22. Takayama S, Watanabe M, Kusuyama H, et al. Evaluation of the effects of acupuncture on blood flow in humans with ultrasound color Doppler imaging. Evid Based Complement Alternat Med. 2012;2012:1-8.

23. Burns PN, Jaffe CC. Quantitative flow measurements with Doppler ultrasound: techniques, accuracy, and limitations. Radiol Clin North Am. 1985;23(4):641-657.

24. Takashi O. Measurement of minimal changes in blood vessels and its application. Journal of Clinical Echocardiography. 2006;7(11): 936-941.

25. [No author] European Glaucoma Society Terminology and Guidelines for Glaucoma, 4th Edition - Chapter 2: Classification and terminology. Br J Ophthalmol. 2017;101(5):73-127.

26. Kearney PM, Whelton M, Reynolds K, Muntner P, Whelton PK, He J. Global burden of hypertension: analysis of worldwide data. Lancet 2005;365(9455):217-223.

27. Costa VP, Harris A, Stefánsson E, et al. The effects of antiglaucoma and systemic medications on ocular blood flow. Prog Retin Eye Res. 2003;22(6):769-805.
Clinical Ophthalmology

\section{Publish your work in this journal}

Clinical Ophthalmology is an international, peer-reviewed journal covering all subspecialties within ophthalmology. Key topics include: Optometry; Visual science; Pharmacology and drug therapy in eye diseases; Basic Sciences; Primary and Secondary eye care; Patien Safety and Quality of Care Improvements. This journal is indexed on

Submit your manuscript here: http://www.dovepress.com/clinical-ophthalmology-journal

\section{Dovepress}

PubMed Central and CAS, and is the official journal of The Society of Clinical Ophthalmology (SCO). The manuscript management system is completely online and includes a very quick and fair peer-review system, which is all easy to use. Visit http://www.dovepress.com/ testimonials.php to read real quotes from published authors. 